\title{
Leveraging blood serotonin as an endophenotype to identify de novo and rare variants involved in autism
}

Rui Chen ${ }^{1,2}$, Lea K. Davis ${ }^{2,3}$, Stephen Guter ${ }^{4}$, Qiang Wei ${ }^{1,2}$, Suma Jacob ${ }^{5}$, Melissa H. Potter ${ }^{1,2}$, Nancy J. Cox ${ }^{2,3}$, Edwin H. Cook ${ }^{4}$, James S. Sutcliffe ${ }^{1,2}$ and Bingshan $\mathrm{Li}^{1,2^{*}}$

\begin{abstract}
Background: Autism spectrum disorder (ASD) is one of the most highly heritable neuropsychiatric disorders, but underlying molecular mechanisms are still unresolved due to extreme locus heterogeneity. Leveraging meaningful endophenotypes or biomarkers may be an effective strategy to reduce heterogeneity to identify novel ASD genes. Numerous lines of evidence suggest a link between hyperserotonemia, i.e., elevated serotonin (5-hydroxytryptamine or 5-HT) in whole blood, and ASD. However, the genetic determinants of blood 5-HT level and their relationship to ASD are largely unknown.
\end{abstract}

Methods: In this study, pursuing the hypothesis that de novo variants (DNVs) and rare risk alleles acting in a recessive mode may play an important role in predisposition of hyperserotonemia in people with ASD, we carried out whole exome sequencing (WES) in 116 ASD parent-proband trios with most (107) probands having 5-HT measurements.

Results: Combined with published ASD DNVs, we identified USP15 as having recurrent de novo loss of function mutations and discovered evidence supporting two other known genes with recurrent DNVs (FOXP1 and KDM5B). Genes harboring functional DNVs significantly overlap with functional/disease gene sets known to be involved in ASD etiology, including FMRP targets and synaptic formation and transcriptional regulation genes. We grouped the probands into High-5HT and Normal-5HT groups based on normalized serotonin levels, and used network-based gene set enrichment analysis (NGSEA) to identify novel hyperserotonemia-related ASD genes based on LoF and missense DNVs. We found enrichment in the High-5HT group for a gene network module (DAWN-1) previously implicated in ASD, and this points to the TGF- $\beta$ pathway and cell junction processes. Through analysis of rare recessively acting variants (RAVs), we also found that rare compound heterozygotes (CHs) in the High-5HT group were enriched for loci in an ASD-associated gene set. Finally, we carried out rare variant group-wise transmission disequilibrium tests (gTDT) and observed significant association of rare variants in genes encoding a subset of the serotonin pathway with ASD.

Conclusions: Our study identified USP15 as a novel gene implicated in ASD based on recurrent DNVs. It also demonstrates the potential value of 5-HT as an effective endophenotype for gene discovery in ASD, and the effectiveness of this strategy needs to be further explored in studies of larger sample sizes.

Keywords: Autism, Autism spectrum disorder, De novo mutation, Rare variants, Compound heterozygotes, 5-HT, Serotonin, Hyperserotonemia, Endophenotype, Whole exome sequencing, Group-wise transmission/disequilibrium test

\footnotetext{
* Correspondence: bingshan.li@vanderbilt.edu

${ }^{1}$ Molecular Physiology \& Biophysics, Vanderbilt University, Nashville, TN, USA

${ }^{2}$ Vanderbilt Genetics Institute, Vanderbilt University, Nashville, TN, USA

Full list of author information is available at the end of the article
}

(c) The Author(s). 2017 Open Access This article is distributed under the terms of the Creative Commons Attribution 4.0 International License (http://creativecommons.org/licenses/by/4.0/), which permits unrestricted use, distribution, and reproduction in any medium, provided you give appropriate credit to the original author(s) and the source, provide a link to the Creative Commons license, and indicate if changes were made. The Creative Commons Public Domain Dedication waiver (http://creativecommons.org/publicdomain/zero/1.0/) applies to the data made available in this article, unless otherwise stated. 


\section{Background}

Autism spectrum disorder (ASD) is an early-onset neurodevelopmental condition with an estimated prevalence of $\sim 1$ in 68 [1]. ASD is expressed across a spectrum of severity in two core phenotypic domains: persistent deficits in social interaction and communication and restricted, repetitive behaviors and interests. ASD is highly heritable, with an estimated heritability of $64-91 \%$ based on a recent meta-analysis [2]. The genetic basis of ASD, however, is complicated by locus heterogeneity for both common allele and rare variant effects. Although common variants in aggregate contribute to a larger proportion $(\sim 50 \%)$ of liability [3], genome-wide association studies (GWAS) with thousands of subjects have not found consistent, strongly associated individual common variants [4-9]. Rare de novo variants, including both copy number (CNVs) and single nucleotide variants $(\mathrm{SNVs})$, play a significant role in ASD liability [10]. To date, dozens of genes harboring de novo CNVs and SNVs meeting genome-wide significance have been identified, and corresponding functional pathways and biological processes have emerged from analysis of these variants [10]. Despite advances in identifying ASD risk loci, major hurdles remain, since rare variants account for only a minority of cases, and effect sizes for common variants necessitate GWAS sample sizes many times those currently available. Data indicate that a thousand or more genes may contribute to ASD liability [11].

In addition to larger samples, another strategy to tackle heterogeneity leverages meaningful endophenotypes or biomarkers that demonstrate heritability $[12,13]$. The hypothesis that endophenotypes reflect variation in a subset of the broader set of disease risk genes leads to the notion that the subgroup of cases that share the endophenotype is more genetically homogeneous. Thus, gene discovery in such a subgroup affords greater power compared with a similarly sized group from the overall disease population and in the case of molecular traits may provide a more direct path to functional mechanisms. Biomarkers and endophenotypes in ASD have drawn interest given the potential to facilitate earlier diagnosis and better prediction of prognosis or treatment response [14].

Hyperserotonemia, or elevated serotonin (5-hydroxytryptamine or 5-HT) in whole blood, is one of the most consistent quantitative traits and biomarkers in ASD since its identification in 1961 [15-18]. In particular, these studies reported a significantly higher 5-HT blood level in about one third of ASD subjects, compared with typically developing controls. The elevated 5-HT level, or hyperserotonemia, is observed in ASD but not in subjects with unspecified intellectual disability [19]. Whole blood 5-HT levels show intermediate elevation in firstdegree relatives of hyperserotonemic probands [20-23]. While hyperserotonemia in ASD shows evidence for heritability, whole blood 5-HT also exhibited high narrow and broad heritability ( 0.51 and 1.0 , respectively) in a Hutterite population sample [24]. Although the mechanism underlying the elevation of serotonin levels in ASD remains unclear, several lines of investigation point to a role for serotonin in ASD etiology [25-29]. In blood, greater than $99 \%$ of the serotonin is stored in platelets, which is taken up from the enterohepatic circulation by the serotonin transporter (SERT), encoded by SLC6A4, after synthesis in enterochromaffin cells of the gut. Linkage studies in ASD have implicated the 17q11.2 region harboring SLC6A4 [30-32]. Hypothesizing rare variants in the absence of significant allelic association at SLC6A4 led to the discovery of multiple functional coding variants [30], and in particular, the association of SERT Ala56 was supported in mice by the evidence that mice carrying the variant displayed alterations in social function, communication, and repetitive behavior and elevated whole blood 5-HT [33]. These findings collectively support hyperserotonemia as a powerful endophenotype for dissecting the genetic etiology of ASD.

In this study, we carried out whole exome sequencing (WES) in a collection of ASD parent-proband trios with 5-HT measurements collected through an Autism Center of Excellence (ACE) study to search for genetic variants implicated in ASD using serotonin as an endophenotype. Given that elevated serotonin was observed in ASD probands compared with their parents, we hypothesize that de novo variants (DNVs) and recessively acting variants (RAVs) play a key role in predisposition of hyperserotonemia and ASD. DNVs observed in probands, but not parents, which disrupt genes involved in 5-HT and ASD, should affect 5-HT levels only in probands; similarly, rare risk alleles acting in a recessive manner (i.e., RAVs) that are transmitted from parents to probands may lead to elevated 5-HT levels in those probands. Both DNVs and RAVs have been implicated in ASD [34]; however, the allelic architecture of hyperserotonemia in autism is unknown. We thus aimed to utilize this unique endophenotype in $\mathrm{ACE}$ trios to identify genes involved in both traits; an approach that we hypothesize effectively reduces genetic heterogeneity. Moreover, we predict that positive findings will shed light on what serotonin-related functional pathways are involved in ASD. Corresponding genes and proteins may offer insights into dysregulated CNS development and point to therapeutic strategies for ASD symptoms.

\section{Methods}

Whole exome sequencing and data processing

Exome capture and sequencing were conducted at the Center for Inherited Disease Research (CIDR) and HudsonAlpha (11 trios). Genomic DNA was extracted 
from either cell line or blood samples collected from all participating family members in ACE. At HudsonAlpha, exons were captured using NimbleGen Seq Cap EZ SR v2 (Roche, Switzerland) and sequenced on an IlluminaHiSeq2000 (Illumina, USA). At CIDR, exons were captured using Agilent SureSelect XT HumanAllExon V5 + UTRs_71MbKit_S04380219 (Agilent Technologies, USA). Sequencing platform and chemistry was Illumina HiSeq2500 (Illumina, USA), TruSeq Rapid PE Cluster Kit-HS (Illumina, USA), and TruSeq Rapid SBS-HS (Illumina, USA) with 100 bp paired-end runs at $\sim 60 \times$. Sequencing reads were aligned to the reference human genome GRCh37 using BWA [35] with default settings. Next, the resulting BAM files were further processed and used for variant calling following the best practice procedures of GATK [36, 37]. We retained biallelic SNVs with PASS VQSR tag and average depth $>10$. We masked genotypes of trios with minimal depth $<6$ as missing. We annotated all variants using ANNOVAR [38] and obtained deleteriousness predictions from nine bioinformatics tools provided in ANNOVAR. Each variant was assigned a deleterious score (DScore) between 0 and 9, denoting the number of damaging predictions from among these algorithms. We used TrioDenovo to call DNVs on both autosomes and X chromosome and obtained reliable candidates for validation after filtering [39]. We focused on two types of functional variants: loss of function (LoF; splice site, nonsense, and frameshift) and missense (Mis) DNVs. We experimentally validated all functional DNVs by Sanger sequencing and obtained 100 validated functional DNVs, including 16 LoF and 84 Mis DNVs across 99 genes (Additional file 1: Table S1).

In RAV analysis, we obtained 2073 LoF and 39,095 Mis-D4 SNVs after filtering. To get reliable RAVs, we used the phase-by-transmission algorithm to construct haplotypes in trios to achieve robust phasing for rare variants [40].

\section{Normalization of whole blood serotonin}

In order to compare whole blood serotonin (WB5HT) values across age and population groups, z-scores were generated to adjust for pubertal status and race/ ethnicity. Pubertal status was determined based on their Tanner stage $[41,42]$ or chronological age. Subjects were classified as pre-pubertal if their Tanner stage was either I or II and post-pubertal if their Tanner stage was greater than or equal to III. In the case of missing Tanner data, chronological age was used to create the puberty variable. Subjects with a chronological age less than 144 months were classified as pre-pubertal, whereas subjects whose chronological age were greater than or equal to 144 months were classified as post-pubertal.
Race and ethnicity information was obtained during an initial screening by parent report. A subject's reported race and ethnicity was classified based on self-report and confirmed by principal components analysis (PCA). Utilizing the respective ancestry group, means and standard deviations (SD) for WB5HT of the pre-pubertal, "normal control" children generated previously, WB5HT z-scores were calculated for our pre-pubertal subjects. For postpubertal subjects, WB5HT z-scores were generated from the WB5HT means and SDs of the adult subjects in [43] (see Additional file 1: Table S2). For subjects with more than one reported race/ethnicity, the average of the respective means and SDs were used to calculate their $\mathrm{z}$ score. Individual subjects taking medications that could potentially influence serotonin as well as other psychotropic medications were excluded from WB5HT analyses.

After normalization, we separated probands into two groups based on the distribution of WB5HT-z in parents and probands (Additional file 2: Figure S1). Specifically, we classified probands with WB5HT-z $>1.75$ as the High-5HT group and probands with WB5HT-z $<1$ as the Normal-5HT group, with 30 and 67 probands in the High-5HT and Normal-5HT groups, respectively. For analysis of WB5HT-z subgroups, we did not include the 10 probands (and corresponding genes) who had values between 1 and 1.75 to increase separation between comparison groups, given the absence of an obvious cut-point for defining hyperserotonemia.

\section{RAV burden in High-5HT group vs. Normal-5HT groups}

For RAV analysis, we carried out PCA on our data combined with the 1000 Genomes Project data and selected probands of Western European descent (25 High-5HT and 38 Normal-5HT) based on the first two PCs to avoid confounds caused by population stratification (Additional file 3: Figure S2). We called two types of RAVs from our data: compound heterozygotes $(\mathrm{CHs})$ and homozygotes. A total of 179 RAVs (137 CH and 42 homozygote genotypes-and corresponding 155 genes-were present in $78 \mathrm{WE}$ probands. Of these RAV genes, 127 are expressed (RPKM $>1$ ) in early fetal brain using data from Brainspan (Brainspan: http:// www.brainspan.org/).

For each gene detected, we carried out the Fisher exact test of RAVs in the High-5HT vs. the Normal-5HT groups. Given the low power to detect significant associations of individual genes, we selected genes with an odds ratio $>1$ as candidates for the risk RAV genes in the High-5HT group and used genes with OR $<1$ as controls.

\section{Gene set enrichment analysis}

We identified several functional/disease gene sets in ASD for gene set enrichment analysis (GSEA): (1) two lists of RBFOX1 (RNA-binding protein, fox-1 homolog 
1)-regulating RNA targets: RBFOX1-1 [44]; RBFOX1-2 [45]; (2) two lists of fragile X mental retardation protein (FMRP) mRNA targets: FMRP-1 [46]; FMRP-2 [47]; (3) evolutionarily constrained genes (ECGs) [48]; (4) synapse-related gene lists: postsynapse-related genes from proteomic profiling of human neocortical biopsies (Hpsd), protein complexes of the postsynaptic density (PSD; PSD-95, ARC, mGluR5, NMDAR), and genes related to presynaptic proteins, presynaptic active zone, and synaptic vesicles $[49,50]$; (5) chromatin remodeling factors (CRFs) [51]; (6) histone modification enzymes (HMEs) [52]; (7) differentially expressed genes from cortical samples of autism (DEs) [44]. All genes including candidates and known gene sets were mapped to current human gene symbols (HGNC) to assure the consistency of nomenclature.

It is well known that, generally, brain-expressed genes tend to be longer, and if a statistic is influenced by gene length, then gene length is a potentially confounding factor for GSEA (Additional file 4: Figure S3). For both DNVs and RAVs, to control for the confounding nature of gene length, we used a sampling scheme to obtain empirical $p$ values. Specifically, we constructed a null distribution of the number of overlapping genes by randomly sampling genes matching the length of the candidate genes and then calculated empirical $p$ values by comparing the observed overlapping gene number to the null distribution. To select a gene with matching length, we sorted genes by their coding sequence (CDS) lengths and randomly picked a gene from the neighbors of target gene. The interval of neighbors is a parameter that may affect the results, e.g., an interval of 30 means that we chose a gene from a subset of genes consisting of 30 shorter and 30 longer neighbors of the target gene. We therefore tested simulated configurations with 30,100, or 1000 neighbors and obtained similar results across all simulated sets. We present the results using an interval of 100 .

\section{Network-based gene set enrichment analysis}

Our approach to the network-based gene set enrichment analysis (NGSEA) is based on the guilt-byassociation principle, in that genes underlying risk for a given disease tend to function together in one or more functional, biological networks. This assumption underlies many approaches that have been successfully applied to search for disease genes in numerous disorders, including ASD [53-55]. Given the extensive heterogeneity of ASD, it is expected that several different networks underlying its etiology exist. To test the hypothesis that a candidate (ACE) gene set is enriched for associated genes, we reframed this hypothesis as being that novel gene candidates identified from our ACE sample are closer to established ASD genes in the network than expected by chance. Accordingly, we devised a network-based approach to measure the distance between two gene sets, similar to EnrichNet [56], and used a sampling scheme to assess the significance. The framework assigns genes in a known ASD gene set as seeds in a functional protein-protein interaction network and then runs label propagation in the network using a random walk with restart $[57,58]$. Briefly, let $A$ be an adjacent matrix representing the gene-gene network, with 1 in the matrix indicating that the corresponding two genes interact and 0 otherwise, $m$ be the number of genes in the network, $F_{0}$ be a vector of length $m$ with the $i$ th element being 1 if the $i$ th gene is a seed gene and 0 otherwise. Label propagation is executed as $F_{\mathrm{t}+1}=(1-\alpha) \times A \times F_{\mathrm{t}}+\alpha \times F_{0}$ until convergence, with $\alpha$ being the restart rate. Following propagation, each value in $F$ represents the influence (i.e., the amount of flow) the corresponding gene receives from the seeds; we termed this influence the propagationbased distance in network (PDN) to measure the distance between a candidate gene and a known ASD gene set (i.e., the seeds). Next, we summed the PDN of all novel candidate genes to a known ASD gene set and used the sum as a measurement of the distance between the candidate set and the known gene set. Finally, we generated pseudo-candidate gene sets by randomly sampling genes from the human genome, controlling for gene length as in the GSEA, and then calculated the sum of the PDN between pseudo sets to the known ASD gene set to build the null distribution; this in turn permits the generation of empirical $p$ values. We used the PINA [59] network in this analysis with a restart probability of 0.3 .

\section{Rare variant group-wise transmission/disequilibrium test}

We collected a set of serotonin-related genes, representing both pre- and postsynaptic functions, including 5-HT metabolism, transport, and signaling (Additional file 1: Table S3). We assigned genes in 5HT receptor family as a group (Receptor group) and genes related to 5-HT metabolism, transport, and other largely presynaptic functions as the other group (Non-Receptor group) due to their distinct mechanisms. We carried out group-wise transmission/disequilibrium test (gTDT) on each of the two groups to test whether rare variants in these genes are associated with ASD. We kept all LoF and Mis-D4 (Dscore $\geq 4$ ) variants with MAF $<0.01$ located on autosomes; in this analysis we used a more stringent criterion of Mis-D4 than Mis-D2 used in DNVs to filter missense variants here to potentially enrich for causal variants. 


\section{Results}

\section{Brief description of samples and sequence data} processing

We sequenced the exomes of 133 trios as a part of the University of Illinois at Chicago ACE project. To avoid confounding our analysis with data from earlier studies, 17 trios that were included in previously published data [60] were excluded, and the remaining 116 trios were used in subsequent analyses. Detailed information on subject ascertainment and clinical assessment has been described elsewhere [61]. For this analysis, one subject with Fragile $\mathrm{X}$ syndrome was excluded but three subjects with clinically significant CNVs $(A 2 B P 1 / R B F O X 1$ deletion [62] $(1.0<$ WB5HT-z $<1.75)$, maternal interstitial duplication 15q11-q13 (Normal-5HT group), and NRXN3 deletion (Normal-5HT group)) were included. Demographic and phenotypic data are summarized in Additional file 1: Table S4. As expected, males were overrepresented with a ratio of 5.44:1 (male:female), which is comparable to the general ASD population [63]. Of the 116 analyzed trios, 107 probands, 72 fathers and 63 mothers had 5-HT measurements. An overview of analyses conducted in this study is presented in Fig. 1.

\section{Analysis of de novo variants Recurrence of LoF DNVs}

We first focused on LoF DNVs as it is well established that rare LoF DNVs can exert large effect sizes on risk of ASD and are more readily detected compared with other types of variants $[11,64]$. Considering the stronger purifying selection conferred by LoF mutations, recurrence of rare LoF DNVs in a specific gene is a strong indicator of disease association, and recurrence of two or more LoF DNVs in a gene typically reaches genome-wide significance for a sample size up to 3000 families [65]. We did not observe genes with recurrent LoF DNVs in our data given the limited sample size. We then compared genes implicated by our DNVs to those from other recent studies, such as the 192 LoF DNVs from the Autism Sequencing Consortium (ASC) [11] and 391 LoF DNVs from the Simons Simplex Collection (SSC) [64]. When combined with previous datasets, our study identified a second LoF DNV in ubiquitinspecific peptidase 15 (USP15) making this the first reported recurrence of LoF DNV in this gene, implicating it as a novel ASD candidate. A frameshift DNV was also identified in FOXP1 (forkhead box P1) and a stop-gain mutation in $K D M 5 B$ (lysine demethylase $5 \mathrm{~B}$ ), adding further support for these two loci already identified as having recurrent, independent DNVs $(N \geq 2)$ within SSC or ASC data. Thus, our studies provide strong support for three loci as high confidence ASD (hcASD) genes based on LoF DNVs.

\section{Enrichment analysis of DNVs in functional gene sets}

We restricted analysis to the genes that are expressed at early stages of brain development (Brainspan, http:// www.brainspan.org/) using a criterion of RPMK $>1$ (16

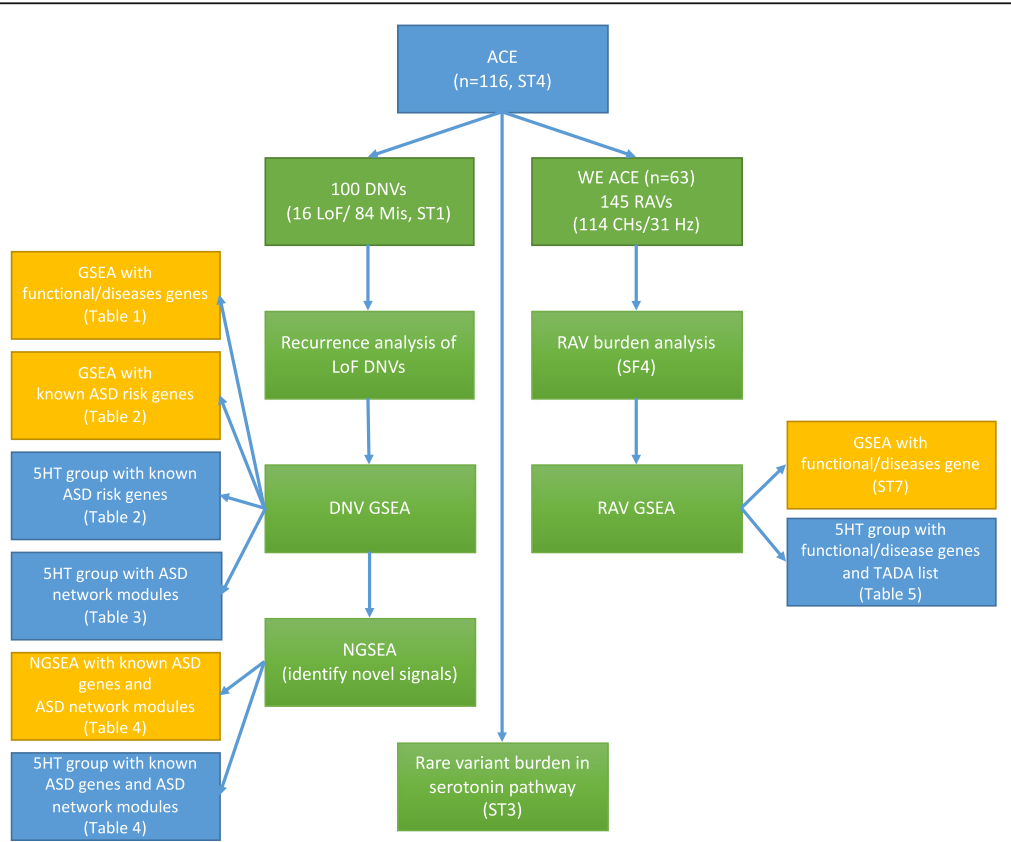

Fig. 1 Overview of the analysis. This study shows the analysis of DNVs and RAVs in 116 families with serotonin measurement. The major steps are shown in the middle (green). The analysis steps identifying ASD-related signals used the whole DNV/RAV list (yellow). The steps identifying 5HT-related signals in ASD used DNVs/RAVs in High-5HT vs. Normal-5HT groups (blue). ACE, Autism Center of Excellence; DNV, de novo variant; RAV, recessive acting variant; LoF, loss of function; GSEA, Gene Set Enrichment Analysis; NGSEA, Network-based Gene Set Enrichment Analysis; 5HT, 5-hydroxytryptamine; TADA, a list from a recent study of both transmitted and de novo variants in ASD [1 1]; $\mathrm{CH}$, compound heterozygote; $\mathrm{Hz}$, homozygote 
LoFs and $70 \mathrm{Mis})$. We first focused on LoF DNVs and the results are shown in Table 1. Note that for the analyses carried out in this study, we report the nominal $p$ values, not corrected for multiple comparisons. We found the most significant enrichment of LoF DNV genes in the HME gene set $(p=0.009)$, of which $K D M 5 B$ is a notable example. LoF DNV loci did not show significant enrichment in the two FMRP target gene sets $(p=0.415 / 0.157)$. FMRP functions as a translational repressor in neurons, and loss of FMRP function causes fragile X syndrome [66-68]. RBFOX1 (previously known as Ataxin-2-binding protein 1, A2BP1) corresponds to the only known splicing factor implicated in ASD, and hemizygous loss of RBFOX1 is associated with ASD and multiple epilepsy syndromes [69]. However, we saw no significant enrichment among its targets $(p=1$ and 0.522). We also did not observe significance enrichment of LoF DNVs in genes under evolutionary constraint (ECGs; $p=0.132$ ) and synaptic protein gene sets.

In testing for enrichment of missense DNV-containing genes with the same functional gene sets, we observed enrichment in FMRP targets and Hpsd (Table 1). When combining missense and LoF DNVs together, significance for FMRP, Hpsd, and HME gene was observed (Table 1). We then combined the LoF DNVs with the SSC and ASC studies to get a comprehensive picture of enrichment in functional/disease gene sets. Consistent results are shown in Additional file 1: Table S5, i.e. RBFOX1-2, FMRP targets, ECGs, Presynaptic genes, and HMEs show strong levels of significance.

Table 1 GSEA of DNVs in functional/disease clusters implicated in ASD (italics: $p<0.05$ )

\begin{tabular}{llll}
\hline & Func (86) & LoFs (16) & Mis (70) \\
\hline RBFOX1-1 (186) & $2(0.342)$ & $0(1)$ & $2(0.318)$ \\
RBFOX1-2 (547) & $5(0.469)$ & $1(0.522)$ & $4(0.542)$ \\
FMRP-1 (936) & $15(0.045)$ & $2(0.415)$ & $13(0.045)$ \\
FMRP-2 (831) & $13(0.136)$ & $3(0.157)$ & $10(0.256)$ \\
ECGs (928) & $10(0.341)$ & $3(0.132)$ & $7(0.598)$ \\
Hpsd (1429) & $15(0.021)$ & $3(0.181)$ & $12(0.027)$ \\
PSD-95 (107) & $1(0.392)$ & $0(1)$ & $1(0.348)$ \\
ARC (25) & $0(1)$ & $0(1)$ & $0(1)$ \\
mGluR5 (37) & $0(1)$ & $0(1)$ & $0(1)$ \\
NMDAR (59) & $0(1)$ & $0(1)$ & $0(1)$ \\
Presynaptic active zone (204) & $1(0.498)$ & $0(1)$ & $1(0.457)$ \\
Presynaptic (330) & $3(0.252)$ & $1(0.239)$ & $2(0.421)$ \\
Vesicles (104) & $1(0.367)$ & $0(1)$ & $1(0.281)$ \\
CRFs (55) & $1(0.358)$ & $0(1)$ & $1(0.361)$ \\
HMEs (146) & $4(0.023)$ & $2(0.009)$ & $2(0.301)$ \\
DEs (411) & $2(0.461)$ & $1(0.268)$ & $1(0.707)$ \\
\hline
\end{tabular}

${ }^{a}$ The number in the header and row names denotes the size of the list. The number of overlapped genes between candidate DNVs and functional/ diseases gene sets and $p$ value (in brackets) are listed in main cells
Gene set enrichment analysis of DNVs in known ASD risk gene sets

Next we investigated to what degree our DNVs identify known ASD risk genes. We collected two ASD gene sets: (1) candidates identified from allelic association studies, rare single gene variants (CNVs and SNVs), and genes linked to syndromic autism, all of which are cataloged in AutDB updated on June, 2015 [70]; (2) genes with recurrent DNVs combining data from ASC and SSC (Recur) $[11,64]$ - we consider the latter to represent a carefully chosen set of hcASD risk genes. Indeed, we found that genes with LoF DNVs are significantly enriched amongst Recur $\left(p=3.817 \times 10^{-4}\right)$ genes (Table 2). The results confirm that LoF DNVs in the ACE trio sample correspond to genes with extant evidence for involvement in ASD risk. In contrast, Mis-D2 DNVs only showed a nonsignificant trend in Recur $(p=0.096)$ and no evidence for enrichment among AutDB genes $(p=0.161)$ (Table 2).

We asked how the signals shown above relate to the distribution of whole blood serotonin by comparing GSEA results of DNV genes in the High-5HT and Normal-5HT groups. Since serotonin level is influenced by several factors like ancestry, age, and pubertal status, we first calculated a normalized z-score for each of the probands, adjusting for ethnicity and age, and assigned to High-5HT or Normal-5HT groups based on a logical split in the distribution ("Methods"). The corresponding gene lists are provided in Additional file 1: Table S6. While the LoF DNVs in the normal-5HT group are significantly enriched in both ASD-associated genes (AutDB: $p=0.036$, Recur: $p=8.989 \times 10^{-5}$ ), LoF DNV genes in the High group showed no such evidence of enrichment (Table 2). Contrasting the Normal-5HT group, we observed no significant enrichment of Mis-D2 DNV genes in the High-5HT group (Table 2).

To further explore our data and map observed DNVs onto biological networks, we tested two gene network constructs previously employed to identify gene networks associated with ASD based on enrichment of rare, damaging variants: three modules (denoted as MAGI-1, 2, 3; for Merging Affected Genes into Integrated networks) reported in [55] and four modules (denoted as

Table 2 GSEA of DNVs with known ASD risk gene sets (italics: $p<0.05$ )

\begin{tabular}{lll}
\hline & AutDB (781) & Recur (37) \\
\hline LoFs (16) & $2(0.124)$ & $2(3.817 e-04)$ \\
Mis-D2 (56) & $4(0.161)$ & $1(0.096)$ \\
High-5HT_LoFs (5) & $0(1.000)$ & $0(1.000)$ \\
High-5HT_Mis-D2 (10) & $0(1.000)$ & $0(1.000)$ \\
Normal-5HT_LoFs (8) & $2(0.036)$ & $2(8.989 e-05)$ \\
Normal-5HT_Mis-D2 (38) & $3(0.179)$ & $1(0.067)$ \\
\hline
\end{tabular}


DAWN-1, 2, 3, 4, for Detecting Association with Networks) reported in [11]. Testing genes with de novo LoF or Mis-D2 variants from High-5HT $(N=5,10)$ or Normal-5HT $(N=8,38)$ groups against the three modules in the MAGI classification, showed no significant enrichment (Table 3). However, using the DAWN modules against DNV loci from the Normal-5HT group, we found significant overlap of LoF genes in DAWN-4 ( $p=$ $\left.9.216 \times 10^{-6}\right)$, Mis-D2 genes in DAWN-1 $(p=0.035)$ and DAWN-3 $\left(p=5.280 \times 10^{-3}\right) \quad$ (Table 3$)$. This suggests that genes harboring functional DNVs from the (major) normal 5-HT part of the ACE cohort are enriched for ASD genes falling into these particular modules. In contrast, no evidence for enrichment was detected for the High-5HT group.

\section{Identification of novel ASD genes through network-based analysis of DNVs}

We were particularly interested in assessing to what degree the novel DNV genes identified in our study ostensibly contribute to ASD risk, which cannot be assessed by GSEA, and thus applied NGSEA to further this goal. We note that NGSEA in this section used the same functional/disease gene sets and network modules, with the exception that the current analyses specifically test for novel signals in network space, while traditional enrichment analysis does not. No enrichment, however, was detected considering either Recur or AutDB sets, or the seven total MAGI/DAWN modules, for both LoF and Mis-D2 DNV genes (Table 4).

Next we aim to inspect whether NGSEA can discover novel signals with the aid of whole blood 5-HT as an endophenotype. Results show that neither group of LoF genes were significantly closer to any of the MAGI modules than expected by chance, with only the exception that Mis-D2 of Normal-5HT group is significantly closer to MAGI-1 (Table 4). We did, however, observe significance for DAWN-1 LoF genes in the High-5HT group ( $p=0.039$, Fig. 2$)$; Normal-5HT LoF loci were not associated with any DAWN modules (Table 4). Relating results from GSEA and NGSEA analyses, data indicate that High-5HT and Normal-5HT LoF genes identify different modules, i.e., High- $5 \mathrm{HT}$ LoF genes identify DAWN-1 using NGSEA (Table 4), and the Normal-5HT
LoF genes detect DAWN-4 using GSEA (Table 3). The complete list of the LoF DNV genes in the High-5HT group is presented in Additional file 1: Table S6 and their enrichment findings for different gene modules are shown in Table 4.

Like with LoF mutations, NGSEA of Mis-D2 DNV genes from the High-5HT group also revealed enrichment with DAWN-1 $(p=0.04$, Fig. 3). However, the Normal-5HT Mis-D2 genes still showed no enrichment to DAWN modules (Table 4). In contrast to the consistent pattern of NGSEA enrichment of High-5HT DNV genes among DAWN-1, genes identified by Normal5 HT DNVs, which showed no enrichment using NGSEA, were enriched in DAWN-1, DAWN-3, and DAWN-4 modules in GSEA.

\section{Analysis of rare recessively acting variants}

In parallel with DNVs, we hypothesize that the elevated 5 -HT in probands compared to their parents is partially accounted for by rare recessively acting variants (RAVs), consisting of compound heterozygotes $(\mathrm{CHs})$ and homozygotes. We focused on functional variants, comprising all LoF and Mis-D2 variants on autosomes with minor allele frequency (MAF) <0.01; we excluded X chromosomes in the RAV analysis due to its inapplicability in males. We used the phase-by-transmission algorithm to construct haplotypes in trios to achieve robust phasing for rare variants [40], and in total, we obtained $137 \mathrm{CHs}$ and 42 homozygotes in probands, distributed in 155 genes. We combined both the $\mathrm{CHs}$ and homozygotes for the analysis of RAVs.

\section{RAV burden associated with ASD}

We first examined whether probands carry a higher burden of RAVs compared to their parents. As the number of RAVs in a given sample and across samples is influenced by sequencing data in complex ways, we normalized functional RAV burden using synonymous RAV burden as a baseline, assuming that synonymous variants do not (by and large) confer ASD risk. Results show that the burden of functional RAVs in probands is not significantly different from that in parents (Additional file 5: Figure S4). We further examined whether genes with RAVs in probands are enriched in functional/

Table 3 GSEA of DNVs in High-5HT and Normal-5HT groups with network modules implicated in ASD (italics: $p<0.05$ )

\begin{tabular}{llllllll}
\hline & MAGI1 (80) & MAGI2 (24) & MAGI3 (91) & DAWN1 (19) & DAWN2 (20) & DAWN3 (58) & DAWN4 (113) \\
\hline LoFs (16) & $0(1.000)$ & $0(1.000)$ & $0(1.000)$ & $0(1.000)$ & $0(1.000)$ & $0(1.000)$ & $3(8.921 e-05)$ \\
Mis-Ds2 (56) & $1(0.199)$ & $0(1.000)$ & $0(1.000)$ & $1(0.051)$ & $0(1.000)$ & $2(0.011)$ & $0(1.000)$ \\
High-5HT_LoFs (5) & $0(1.000)$ & $0(1.000)$ & $0(1.000)$ & $0(1.000)$ & $0(1.000)$ & $0(1.000)$ & $0(1.000)$ \\
High-5HT_Mis-D2(10) & $0(1.000)$ & $0(1.000)$ & $0(1.000)$ & $0(1.000)$ & $0(1.000)$ & $0(1.000)$ & $0(1.000)$ \\
Normal-5HT_LoFs (8) & $0(1.000)$ & $0(1.000)$ & $0(1.000)$ & $0(1.000)$ & $0(1.000)$ & $0(1.000)$ & $3(9.216 e-06)$ \\
Normal-5HT_Mis-D2 (38) & $1(0.140)$ & $0(1.000)$ & $0(1.000)$ & $1(0.035)$ & $0(1.000)$ & $2(5.280 e-03)$ & $0(1.000)$ \\
\hline
\end{tabular}


Table 4 NGSEA to identify novel signals in different groups of DNVs (italics: $p<0.05$ )

\begin{tabular}{llllllllll}
\hline & AutDB & Recur & MAGI1 & MAGI2 & MAGI3 & DAWN1 & DAWN2 & DAWN3 & DAWN4 \\
\hline LoFs & 0.838 & 0.07 & 0.225 & 0.326 & 0.224 & 0.187 & 0.52 & 0.502 & 0.748 \\
Mis-D2 & 0.762 & 0.135 & 0.026 & 0.347 & 0.371 & 0.083 & 0.274 & 0.353 & 0.055 \\
High-5HT_Func & 0.734 & 0.467 & 0.178 & 0.759 & 0.83 & 0.027 & 0.335 & 0.376 \\
High-5HT_LoFs & 0.925 & 0.234 & 0.746 & 0.713 & 0.637 & 0.039 & 0.653 & 0.82 & 0.244 \\
Normal-5HT_LoFs & 0.481 & 0.046 & 0.139 & 0.147 & 0.104 & 0.33 & 0.801 & 0.233 \\
High-5HT_Mis-D2 & 0.552 & 0.512 & 0.098 & 0.637 & 0.748 & 0.04 & 0.187 & 0.235 & 0.506 \\
Normal-5HT_Mis-D2 & 0.612 & 0.1 & 0.031 & 0.249 & 0.25 & 0.174 & 0.207 & 0.329 & 0.035 \\
\hline
\end{tabular}

disease gene sets in ASD. After correcting for gene length, RAV gene sets showed no evidence to support enrichment amongst the various functional/disease gene sets (Additional file 1: Table S7), likely due to some combination of small sample size and predicted weaker effects of RAVs (relative to DNVs) in conferring ASD risk.

\section{RAVs associated with hyperserotonemia}

We applied GSEA to selected functional RAVs that, we envisioned, are more likely to be enriched in the High5 HT group ("Methods") on the same functional/disease gene sets, correcting for the gene length bias. We observed no significant enrichment among any gene sets, except a trend $(p=0.051)$ towards significance in FMRP targets (Table 5). We then used a gene list corresponding to loci from a recent study of both transmitted and de novo variants in ASD [11]. Termed TADA-1, this analysis from the Autism Sequencing Consortium

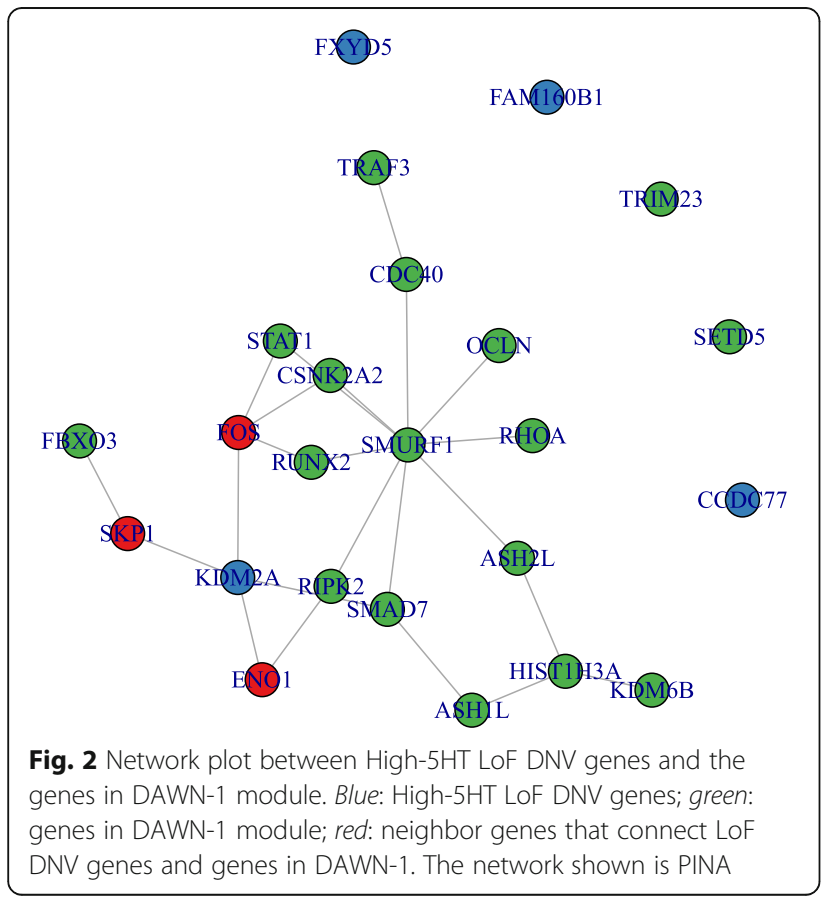

identified a set of 33 hcASD risk loci. Using this gene set, we observed significant enrichment for RAVcontaining genes from this ACE sample $(p=0.03)$ (Table 5). The significance level is not as striking as that observed for DNVs (Table 1), likely owing to the smaller sample size of the WE subset of ACE and the weaker effect of RAVs compared to those from DNVs. Two genes, electron transfer flavoprotein beta subunit $(E T F B)$ and reelin $(R E L N)$, from the TADA-1 comparison were found amongst the RAV gene set, and this remained significant after correction for gene length. We also carried out GSEA with a list termed TADA-2, an extended list of 65 hcASD risk loci (63 are expressed at early stages of brain development) which integrated small de novo deletions with data of TADA-1 [10]. We did not observe significance in this gene set $(p=0.34)$, with a possible reason that the gene RELN, which is one of the two overlapping genes in 33 TADA- 1 hcASD risk genes, is not included in the 65 TADA- 2 genes. Of note, RELN has been implicated in ASD based on numerous studies

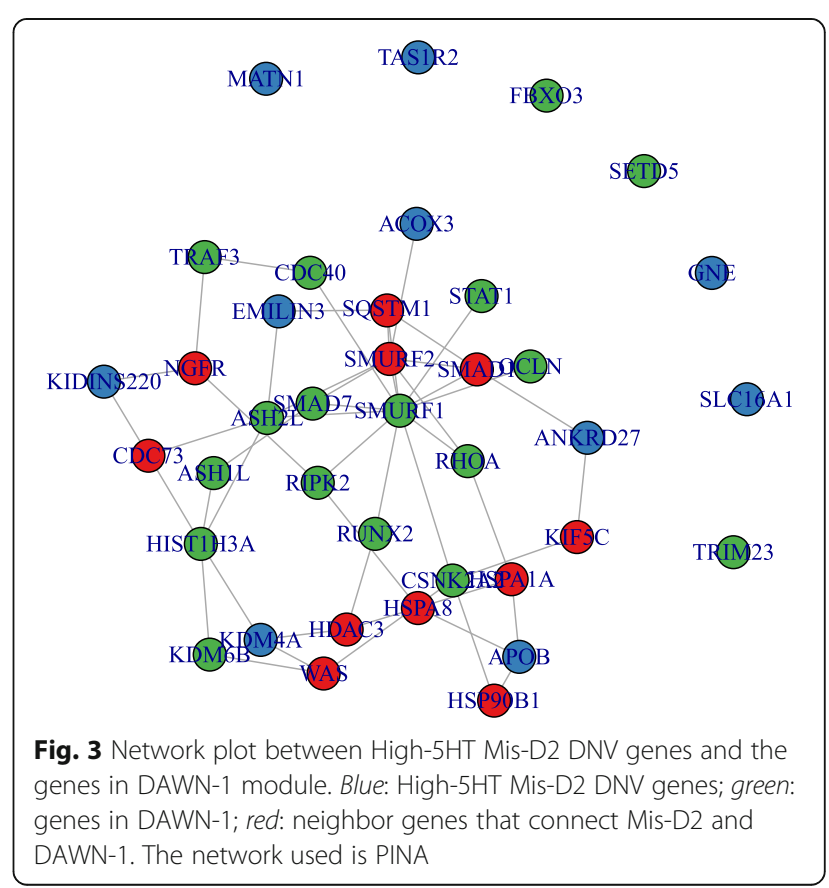


Table 5 GSEA of RAVs in the High-5HT group before and after correcting for gene length (italics: $p<0.05$ )

\begin{tabular}{lll}
\hline & Before correction & After correction \\
\hline RBFOX1-1 (186) & $1(0.413)$ & 0.5 \\
RBFOX1-2 (547) & $3(0.204)$ & 0.57 \\
FMRP-1 (936) & $5(0.126)$ & 0.68 \\
FMRP-2 (831) & $10(9.108 e-05)$ & 0.051 \\
ECGs (928) & $3(0.497)$ & 0.95 \\
Hpsd (1429) & $5(0.385)$ & 0.46 \\
PSD-95 (107) & $0(1.000)$ & 1 \\
ARC (25) & $0(1.000)$ & 1 \\
mGluR5 (37) & $1(0.100)$ & 0.18 \\
NMDAR (59) & $0(1.000)$ & 1 \\
Presynaptic active zone (204) & $0(1.000)$ & 1 \\
Presynaptic (330) & $2(0.242)$ & 0.24 \\
Vesicles (104) & $0(1.000)$ & 1 \\
CRFs (55) & $1(0.145)$ & 0.26 \\
HMEs (146) & $0(1.000)$ & 1 \\
DEs (411) & $1(0.695)$ & 0.57 \\
TADA-1 (33) & $2(3.958 \mathrm{e}-03)$ & 0.03 \\
TADA-2 (63) & $1(0.165)$ & 0.34 \\
\hline
\end{tabular}

[71]. As a control, we also performed GSEA on the RAVs that are enriched in Normal-5HT group ("Methods"), and there is no enrichment in all functional/disease gene sets (Additional file 1: Table S7). We further carried out network-based enrichment analysis of the candidate RAV genes, using the same two sets of network modules and did not observe the enrichment patterns observed for DNVs.

\section{RAV burden in serotonin pathway genes}

We then tested whether rare variants in serotonin pathway genes were associated with ASD by carrying out the gTDT. Because receptors represent one major category of proteins, we elected to select this functional axis as the basis for subdividing 5-HT-related genes for tests of transmission. Thus, we tested 5HT-receptor genes as one group and the remaining genes as the Non-Receptor group ("Methods"). We observed a significant over-transmission of rare functional alleles in the Non-Receptor group of genes (Transmitted: Non-Transmission $=26: 13, p=0.042$ ), which largely encode presynaptic proteins (e.g., SLC6A4, $I T G B 3)$. In contrast to functional variants, synonymous variants exhibit approximately equal transmissions, consistent with the null expectation $(62: 60, p=0.865)$. Functional variants in the Receptor group showed significant under-transmission (1:9, $p=0.021)$, although the total allele count is small. In contrast, there is no evidence for transmission distortion in the Receptor group for synonymous variants $(28: 36, p=0.346)$. We also carried out gTDT on the gene set combining both Receptor and Non-Receptor groups and did not observe significance $(p=0.484)$.

\section{Discussion}

ASD is a genetically heterogeneous disorder with estimates of 1000 or more genes involved in disease etiology. This heterogeneity poses great challenges to identify individually significant risk loci. This challenge is particularly pronounced for DNVs, as mutation rates are extremely low to observe independent de novo mutations in the same gene in a given cohort. LoF DNVs in ASD probands, although rare, are likely to have large effects when predisposing ASD risk, and therefore more likely to identify risk genes. Accordingly, when recurrence of LoF DNM in the same genes is seen in a cohort of probands, it is a strong indicator of that gene's contribution to ASD risk. In this study, we have a very limited sample size compared to other consortium-level datasets, and unsurprisingly did not observe recurrent/independent DNVs in a gene within our data. Instead, we combined our DNVs with those from previous studies for recurrence analysis. We identified one new recurrent gene, USP15, as a novel ASD candidate gene, and provided further supporting evidence for two other known recurrent DNV genes (FOXP1 and KDM5B). FOXP1 has been linked to several cognitive disorders, and its deletion causes autism-like behaviors in mice [72]. KDM5B harbored LoF DNVs in each of two other study cohorts (two in SSC, one in ASC), and probands with KDM5B LoF DNVs were shown to have lower non-verbal IQ [64]. We note that de novo LoF DNVs in KDM5B were also observed in two unaffected (unrelated) siblings in the SSC, suggesting incomplete penetrance. USP15 acts as deubiquitinating enzyme on transforming growth factor-beta (TGF- $\beta$ ) and bone morphogenetic protein (BMP) stimulated R-SMADs (receptor-regulated intracellular proteins that transduce extracellular signals). We note that both TGF- $\beta$ and BMP signaling are involved in differentiation of serotonergic neurons [73], but the role of USP15 in ASD is unclear. With accumulating ASD exome or whole genome sequencing being made public, leveraging previously reported DNVs is an effective strategy for clearly establishing the role of novel risk genes in ASD.

In this study, we implemented several approaches to tackle heterogeneity. First, we separated established ASD genes into network modules that likely represent more homogenous functions. The second was to leverage 5HT as an endophenotype.

Genetic variants implicated in both hyperserotonemia and ASD are enriched in the subset of probands with hyperserotonemia so that we are equipped with increased power to detect ASD genes that function 
through regulating serotonin levels. Using this strategy in our data, we were able to identify novel candidate ASD genes not identified in previous large-scale studies that (we imagine) might poorly represent "hyperserotonemic ASD" risk factors. Although the significance is not striking in NGSEA, the non-overlapping LoF and Mis-D2 genes in the High-5HT group show enrichment in the same module (DAWN-1). In contrast, the genes harboring functional DNVs in the Normal-5HT group did not uncover new ASD genes in NGSEA, probably due to that fact that the majority of ASD patients have normal 5-HT, so that genes identified in previous large-scale studies are already more likely to represent the genes identified in the Normal-5HT probands studied here.

In this study we focused on DNVs and RAVs, two mechanisms that we hypothesized are involved in serotonin-related ASD genetic etiology. It is evident that signals due to DNVs are noticeably stronger than RAVs, presumably due in part to the larger effect sizes for DNVs and in part to the need to restrict analysis of RAVs to those from European subjects. For RAVs we observed significant enrichment with the TADA-1 list, which was derived by TADA's joint modeling of both de novo and inherited variants in a previous study [11]. We analyzed homozygotes and CHs separately, and it is the $\mathrm{CHs}$, not homozygotes, that showed significant enrichment. For example, all of the RAV genes overlapping with the TADA list are $\mathrm{CHs}$, among which two genes (ETFB and RELN) are in the High-5HT group and two, a lysine methyltransferase and a calcium channel gene, are in the Normal-5HT group (CACNA2D3 and KMT2C).

We note that the sample size of our study is small given the context of extensive genetic heterogeneity of ASD. For all association and enrichment analyses, we reported nominal $p$ values without correcting for multiple testing. Varying degrees of dependency across tests makes adjustment for multiple comparisons a challenging problem, even when simulations are used to estimate significance empirically. Given the overall enrichment patterns in biologically relevant gene sets and pathways, our analyses provide promising candidates for further validation in large-scale studies.

\section{Conclusions}

Our study identified novel ASD genes and provided further support for previously reported genes harboring DNVs implicated in ASD. Leveraging 5- $\mathrm{HT}$ as an endophenotype, we identified novel candidate genes related to the TGF- $\beta$ pathway and cell junction function as contributing to serotonin-related ASD risk. Our study demonstrated the value of 5HT as an effective endophenotype in identification of novel ASD genes, warranting the collection of 5-HT in probands for future ASD genetic studies.

\section{Additional files}

Additional file 1: Table S1. List of DNVs identified in our data. Table S2. Mean and standard deviation used for normalization of WB5HT. Table S3. Serotonin pathway genes. Table S4. Summary of subject demographics and phenotype data. Table S5. GSEA of LoF DNVs in ASC, SSC and ACE. Table S6. Gene lists disrupted by different types of DNVs in the High-5HT and the Normal-5HT groups. Table S7. GSEA of all RAVs and Normal-5HT RAVs. (XLSX $47 \mathrm{~kb}$ )

Additional file 2: Figure S1. The distribution of normalized $5-\mathrm{HT}$ in parents and probands. The two vertical lines at 5-HT=1.0 and 1.75 are the cutoffs used to define the High group $(5 \mathrm{HT}>1.75)$ and the Normal$5 \mathrm{HT}$ group (5HT < 1.0). (PDF $19 \mathrm{~kb}$ )

Additional file 3: Figure S2. The result of PCA indicates the threshold to identify the individuals with European ancestry. Left panel: PC plot of CEU, CHB, YRI from the 1000 Genome Project. Right panel: PC plot of our data. (PDF $8 \mathrm{~kb}$ )

Additional file 4: Figure S3. Gene length distribution of functional/ disease gene sets used in GSEA. Most of gene sets have higher median gene length than the set of all genes (background distribution). The red line indicates the median length of all genes in the genome. (PDF $80 \mathrm{~kb}$ )

Additional file 5: Figure S4. Burden of RAVs in Fathers, Mothers and Probands. (a) All RAVs. (b) Homozygous RAVs. (c) Compound heterozygous RAVs. (PNG $430 \mathrm{~kb}$ )

\section{Abbreviations}

5-HT: 5-Hydroxytryptamine; ACE: Autism Center of Excellence; ASC: Autism Sequencing Consortium; ASD: Autism spectrum disorder; CDS: Coding sequence; $\mathrm{CH}$ : Compound heterozygote; CIDR: Center for Inherited Disease Research; CNS: Central nervous system; CNV: Copy number variant; CRF: Chromatin remodeling factors; DAWN: Detecting Association with Networks; DEs: Differentially expressed genes; DNV: De novo variant; Dscore: Deleteriousness score; ECG: Evolutionarily constrained gene; FMRP: Fragile X mental retardation protein; GSEA: Gene set enrichment analysis; GWAS: Genome-wide association studies; hcASD gene: High confidence ASD gene; High-5HT: Group with 5-HT >1.75; HME: Histone modification enzyme; Hpsd: Postsynapse-related genes from proteomic profiling of human neocortical biopsies; LoF: Loss of function; MAGl: Merging Affected Genes into Integrated networks; Mis: Missense; Mis-D2: Missense variants with Dscore $\leq 2$; NGSEA: Network-based gene set enrichment analysis; Normal-5HT: Group with 5-HT <1; PDN: Propagation-based Distance in Networks; PSD: Postsynaptic density; RAV: Recessively acting variant; RBFOX1: RNA-binding protein, fox-1 homolog 1; SNV: Single nucleotide variant; SSC: Simons Simplex Collection; WES: Whole exome sequencing

\section{Acknowledgements}

Expert technical assistance was provided by Kathleen Hennessy, Kelley Moore, and Zengping Hao. We would like to thank the individuals with ASD and their family members for their participation.

\section{Funding}

The UIC Autism Center of Excellence was supported by P50HD055751 (LD, SJ, NC, EC, JS) from NICHD/NINDS/NIEHS. Sequencing services were provided by the Center for Inherited Disease Research (CIDR) through X01 HG007235. CIDR is fully funded through a federal contract from the National Institutes of Health to The Johns Hopkins University, contract number HHSN268201200008l. The study was also supported by R01 MH089482 (JS), P50MH094267 (EC, NC) and a Lever Award from the Chicago Biomedical Consortium. The analysis of the data was supported by R01HG006857 (RC, QW, and BL).

\section{Availability of data and materials}

The datasets generated and/or analyzed during the current study are available in the dbGaP repository under dbGaP study accession phs000712.v1.p1 (http:// www.ncbi.n/m.nih.gov/projects/gap/cgi-bin/study.cgi?study_id=phs000712. v1.p1), and at the National Database for Autism Research: NDARCOL0000001, Study \#416 (http://dx.doi.org/10.15154/1271285). 


\section{Authors' contributions}

RC, LD, NC, EC, JS, and BL conceived and designed the study. RC carried out the major analyses. LD and QW contributed to data processing. SG, SJ, and MP managed patient samples and clinical data. EC, JS, and BL supervised the study. RC, SD, EC, JS, and BL wrote the manuscript. All authors read and approved the final manuscript.

\section{Competing interests}

The authors declare that they have no competing interests.

\section{Consent for publication}

Not applicable.

\section{Ethics approval and consent to participate}

A description of the study was provided to all participants prior to obtaining written informed consent. The study and consent procedures were approved by the University of Illinois at Chicago IRB (protocol\# 2007-0239) and by the Vanderbilt IRB (protocol\# 010143).

\section{Publisher's Note}

Springer Nature remains neutral with regard to jurisdictional claims in published maps and institutional affiliations.

\section{Author details}

${ }^{1}$ Molecular Physiology \& Biophysics, Vanderbilt University, Nashville, TN, USA. ${ }^{2}$ Vanderbilt Genetics Institute, Vanderbilt University, Nashville, TN, USA. ${ }^{3}$ Division of Genetic Medicine, Department of Medicine, Vanderbilt University, Nashville, TN, USA. ${ }^{4}$ Department of Psychiatry, University of Illinois at Chicago, Chicago, IL, USA. ${ }^{5}$ Department of Psychiatry, University of Minnesota, Minneapolis, MN, USA.

Received: 30 August 2016 Accepted: 10 March 2017

Published online: 21 March 2017

\section{References}

1. Christensen DL, Baio J, Braun KV, Bilder D, Charles J, Constantino JN, Daniels J, Durkin MS, Fitzgerald RT, Kurzius-Spencer M, et al. Prevalence and characteristics of autism spectrum disorder among children aged 8 years autism and developmental disabilities monitoring network, 11 sites, United States, 2012. MMWR Surveill Summ. 2016;65:1-23.

2. Tick B, Bolton P, Happe F, Rutter M, Rijsdijk F. Heritability of autism spectrum disorders: a meta-analysis of twin studies. J Child Psychol Psychiatry. 2016; 57:585-95.

3. Klei L, Sanders SJ, Murtha MT, Hus V, Lowe JK, Willsey AJ, Moreno-De-Luca D, Yu TW, Fombonne E, Geschwind D, et al. Common genetic variants, acting additively, are a major source of risk for autism. Mol Autism. 2012;3:9.

4. Wang K, Zhang HT, Ma DQ, Bucan M, Glessner JT, Abrahams BS, Salyakina D, Imielinski M, Bradfield JP, Sleiman PMA, et al. Common genetic variants on 5p14.1 associate with autism spectrum disorders. Nature. 2009:459:528-33.

5. Ma DQ, Salyakina D, Jaworski JM, Konidari I, Whitehead PL, Andersen AN, Hoffman JD, Slifer SH, Hedges DJ, Cukier HN, et al. A genome-wide association study of autism reveals a common novel risk locus at 5 p14.1. Ann Hum Genet. 2009;73:263-73.

6. Weiss LA, Arking DE, Consortium JHA. A genome-wide linkage and association scan reveals novel loci for autism. Nature. 2009;461:802-U862.

7. Salyakina D, Ma DQ, Jaworski JM, Konidari I, Whitehead PL, Henson R, Martinez D, Robinson $J$, Sacharow S, Wright HH, et al. Variants in several genomic regions associated with asperger disorder. Autism Res. 2010;3:303-10.

8. Hussman JP, Chung RH, Griswold AJ, Jaworski JM, Salyakina D, Ma DQ, Konidari I, Whitehead PL, Vance JM, Martin ER, et al. A noise-reduction GWAS analysis implicates altered regulation of neurite outgrowth and guidance in autism. Mol Autism. 2011;2(1):1.

9. Anney R, Klei L, Pinto D, Regan R, Conroy J, Magalhaes TR, Correia C, Abrahams BS, Sykes N, Pagnamenta AT, et al. A genome-wide scan for common alleles affecting risk for autism. Hum Mol Genet. 2010;19:4072-82.

10. Sanders SJ, Xin H, Willsey AJ, Ercan-Sencicek AG, Samocha KE, Cicek AE, Murtha MT, Bal VH, Bishop SL, Shan D, et al. Insights into autism spectrum disorder genomic architecture and biology from 71 Risk Loci. Neuron. 2015; $87: 1215-33$
11. De Rubeis S, He X, Goldberg AP, Poultney CS, Samocha K, Cicek AE, Kou Y, Liu L, Fromer M, Walker S, et al. Synaptic, transcriptional and chromatin genes disrupted in autism. Nature. 2014;515:209-15.

12. Gottesman II, Gould TD. The endophenotype concept in psychiatry: etymology and strategic intentions. Am J Psychiatry. 2003;160:636-45.

13. Ruggeri B, Sarkans U, Schumann G, Persico AM. Biomarkers in autism spectrum disorder: the old and the new. Psychopharmacology (Berl). 2014; 231:1201-16.

14. Walsh $P$, Elsabbagh $M$, Bolton $P$, Singh I. In search of biomarkers for autism: scientific, social and ethical challenges. Nat Rev Neurosci. 2011;12:603-12.

15. Katsui T, Okuda M, Usuda S, Koizumi T. Kinetics of $3 H$-serotonin uptake by platelets in infantile autism and developmental language disorder (including five pairs of twins). J Autism Dev Disord. 1986;16:69-76.

16. Cook EH, Leventhal BL. The serotonin system in autism. Curr Opin Pediatr. 1996:8:348-54.

17. Gabriele S, Sacco R, Persico AM. Blood serotonin levels in autism spectrum disorder: a systematic review and meta-analysis. Eur Neuropsychopharmacol. 2014;24:919-29.

18. Schain RJ, Freedman DX. Studies on 5-hydroxyindole metabolism in autistic and other mentally retarded children. J Pediatr. 1961;58:315-20.

19. Mulder EJ, Anderson GM, Kema IP, de Bildt A, van Lang ND, den Boer JA, Minderaa RB. Platelet serotonin levels in pervasive developmental disorders and mental retardation: diagnostic group differences, within-group distribution, and behavioral correlates. J Am Acad Child Adolesc Psychiatry. 2004:43:491-9.

20. Abramson RK, Wright HH, Carpenter R, Brennan W, Lumpuy O, Cole E, Young SR. Elevated blood serotonin in autistic probands and their 1stdegree relatives. J Autism Dev Disord. 1989;19:397-407.

21. Leboyer M, Philippe A, Bouvard M, Guilloud-Bataille M, Bondoux D, Tabuteau F, Feingold J, Mouren-Simeoni MC, Launay JM. Whole blood serotonin and plasma beta-endorphin in autistic probands and their firstdegree relatives. Biol Psychiatry. 1999:45:158-63.

22. Piven J, Palmer P. Psychiatric disorder and the broad autism phenotype: evidence from a family study of multiple-incidence autism families. Am J Psychiatry. 1999;156:557-63.

23. Cook EH, Arora RC, Anderson GM, Berrykravis EM, Yan SY, Yeoh HC, Sklena PJ, Charak DA, Leventhal BL. Platelet serotonin studies in hyperserotonemic relatives of children with autistic disorder. Life Sci. 1993;52:2005-15.

24. Abney M, McPeek MS, Ober C. Broad and narrow heritabilities of quantitative traits in a founder population. Am J Hum Genet. 2001;68:1302-7.

25. Chugani DC, Muzik O, Rothermel R, Behen M, Chakraborty P, Mangner T, da Silva EA, Chugani HT. Altered serotonin synthesis in the dentatothalamocortical pathway in autistic boys. Ann Neurol. 1997;42:666-9

26. Chugani DC, Muzik O, Behen M, Rothermel R, Janisse JJ, Lee J, Chugani HT. Developmental changes in brain serotonin synthesis capacity in autistic and nonautistic children. Ann Neurol. 1999;45:287-95.

27. Chugani DC. Role of altered brain serotonin mechanisms in autism. Mol Psychiatry. 2002;7 Suppl 2:S16-17

28. McDougle CJ, Naylor ST, Cohen DJ, Aghajanian GK, Heninger GR, Price LH. Effects of tryptophan depletion in drug-free adults with autistic disorder. Arch Gen Psychiatry. 1996;53:993-1000.

29. Yang CJ, Tan HP, Du YJ. The developmental disruptions of serotonin signaling may involved in autism during early brain development. Neuroscience. 2014;267:1-10

30. Sutcliffe JS, Delahanty RJ, Prasad HC, McCauley JL, Han Q, Jiang L, Li C, Folstein SE, Blakely RD. Allelic heterogeneity at the serotonin transporter locus (SLC6A4) confers susceptibility to autism and rigid-compulsive behaviors. Am J Hum Genet. 2005;77:265-79.

31. Stone JL, Merriman B, Cantor RM, Yonan AL, Gilliam TC, Geschwind DH, Nelson SF. Evidence for sex-specific risk alleles in autism spectrum disorder. Am J Hum Genet. 2004;75:1117-23.

32. Cantor RM, Kono N, Duvall JA, Alvarez-Retuerto A, Stone JL, Alarcon M, Nelson SF, Geschwind DH. Replication of autism linkage: fine-mapping peak at 17q21. Am J Hum Genet. 2005:76:1050-6.

33. Veenstra-VanderWeele J, Muller CL, Iwamoto H, Sauer JE, Owens WA, Shah CR Cohen J, Mannangatti $\mathrm{P}$, Jessen T, Thompson BJ, et al. Autism gene variant causes hyperserotonemia, serotonin receptor hypersensitivity, social impairment and repetitive behavior. Proc Natl Acad Sci U S A. 2012:109:5469-74.

34. Morrow EM, Yoo SY, Flavell SW, Kim TK, Lin Y, Hill RS, Mukaddes NM, Balkhy S, Gascon G, Hashmi A, et al. Identifying autism loci and genes by tracing recent shared ancestry. Science. 2008;321:218-23. 
35. Li H, Durbin R. Fast and accurate short read alignment with BurrowsWheeler transform. Bioinformatics. 2009;25:1754-60.

36. McKenna A, Hanna M, Banks E, Sivachenko A, Cibulskis K, Kernytsky A, Garimella K, Altshuler D, Gabriel S, Daly M, DePristo MA. The Genome Analysis Toolkit: a MapReduce framework for analyzing next-generation DNA sequencing data. Genome Res. 2010;20:1297-303.

37. DePristo MA, Banks E, Poplin R, Garimella KV, Maguire JR, Hartl C, Philippakis AA, del Angel G, Rivas MA, Hanna M, et al. A framework for variation discovery and genotyping using next-generation DNA sequencing data. Nat Genet. 2011;43:491-8.

38. Wang K, Li M, Hakonarson H. ANNOVAR: functional annotation of genetic variants from high-throughput sequencing data. Nucleic Acids Res. 2010;38:e164.

39. Wei Q, Zhan X, Zhong X, Liu Y, Han Y, Chen W, Li B. A Bayesian framework for de novo mutation calling in parents-offspring trios. Bioinformatics. 2015; 31:1375-81.

40. Chen R, Wei Q, Zhan X, Zhong X, Sutcliffe JS, Cox NJ, Cook EH, Li C, Chen W, Li B. A haplotype-based framework for group-wise transmission/ disequilibrium tests for rare variant association analysis. Bioinformatics. 2015; 31:1452-9.

41. Marshall WA, Tanner JM. Variations in pattern of pubertal changes in girls. Arch Dis Child. 1969:44:291-303.

42. Marshall WA, Tanner JM. Variations in the pattern of pubertal changes in boys. Arch Dis Child. 1970;45:13-23.

43. McBride PA, Anderson GM, Hertzig ME, Snow ME, Thompson SM, Khait VD, Shapiro T, Cohen DJ. Effects of diagnosis, race, and puberty on platelet serotonin levels in autism and mental retardation. J Am Acad Child Adolesc Psychiatry. 1998;37:767-76.

44. Voineagu I, Wang X, Johnston P, Lowe JK, Tian Y, Horvath S, Mill J, Cantor RM, Blencowe BJ, Geschwind DH. Transcriptomic analysis of autistic brain reveals convergent molecular pathology. Nature. 2011:474:380-4.

45. Zhang C, Zhang Z, Castle J, Sun S, Johnson J, Krainer AR, Zhang MQ Defining the regulatory network of the tissue-specific splicing factors Fox- 1 and Fox-2. Genes Dev. 2008;22:2550-63.

46. Ascano Jr M, Mukherjee N, Bandaru P, Miller JB, Nusbaum JD, Corcoran DL, Langlois C, Munschauer M, Dewell S, Hafner M, et al. FMRP targets distinct mRNA sequence elements to regulate protein expression. Nature. 2012;492: 382-6.

47. Darnell JC, Van Driesche SJ, Zhang C, Hung KY, Mele A, Fraser CE, Stone EF, Chen C, Fak JJ, Chi SW, et al. FMRP stalls ribosomal translocation on mRNAs linked to synaptic function and autism. Cell. 2011;146:247-61.

48. Samocha KE, Robinson EB, Sanders SJ, Stevens C, Sabo A, McGrath LM, Kosmicki JA, Rehnstrom K, Mallick S, Kirby A, et al. A framework for the interpretation of de novo mutation in human disease. Nat Genet. 2014; 46(9):944-50.

49. Bayes A, van de Lagemaat LN, Collins MO, Croning MD, Whittle IR, Choudhary JS, Grant SG. Characterization of the proteome, diseases and evolution of the human postsynaptic density. Nat Neurosci. 2011;14:19-21.

50. Pirooznia M, Wang T, Avramopoulos D, Valle D, Thomas G, Huganir RL, Goes FS, Potash JB, Zandi PP. SynaptomeDB: an ontology-based knowledgebase for synaptic genes. Bioinformatics. 2012;28:897-9.

51. Shipra A, Chetan K, Rao MR. CREMOFAC - a database of chromatin remodeling factors. Bioinformatics. 2006;22:2940-4.

52. Khare SP, Habib F, Sharma R, Gadewal N, Gupta S, Galande S. HIstome-a relational knowledgebase of human histone proteins and histone modifying enzymes. Nucleic Acids Res. 2012;40:D337-342.

53. Parikshak NN, Luo R, Zhang A, Won H, Lowe JK, Chandran V, Horvath S, Geschwind DH. Integrative functional genomic analyses implicate specific molecular pathways and circuits in autism. Cell. 2013;155:1008-21.

54. Willsey AJ, Sanders SJ, Li M, Dong S, Tebbenkamp AT, Muhle RA, Reilly SK, Lin L, Fertuzinhos S, Miller JA, et al. Coexpression networks implicate human midfetal deep cortical projection neurons in the pathogenesis of autism. Cell. 2013:155:997-1007.

55. Hormozdiari F, Penn O, Borenstein E, Eichler EE. The discovery of integrated gene networks for autism and related disorders. Genome Res. 2015;25:142-54.

56. Glaab E, Baudot A, Krasnogor N, Schneider R, Valencia A. EnrichNet: network-based gene set enrichment analysis. Bioinformatics. 2012;28:1451-7.

57. Can T, Çamoǧlu O, Singh AK. Analysis of protein-protein interaction networks using random walks. In: Proceedings of the 5th international workshop on Bioinformatics. Chicago: ACM; 2005. p. 61-8.

58. Chipman KC, Singh AK. Predicting genetic interactions with random walks on biological networks. BMC Bioinformatics. 2009;10:17.
59. Cowley MJ, Pinese M, Kassahn KS, Waddell N, Pearson JV, Grimmond SM, Biankin AV, Hautaniemi S, Wu J. PINA v2.0: mining interactome modules. Nucleic Acids Res. 2012;40:D862-865.

60. Neale BM, Kou Y, Liu L, Ma'ayan A, Samocha KE, Sabo A, Lin CF, Stevens C, Wang LS, Makarov $V$, et al. Patterns and rates of exonic de novo mutations in autism spectrum disorders. Nature. 2012;485:242-5.

61. Levin-Decanini T, Maltman N, Francis SM, Guter S, Anderson GM, Cook EH, Jacob S. Parental broader autism subphenotypes in ASD affected families: relationship to gender, child's symptoms, SSRI treatment, and platelet serotonin. Autism Res. 2013;6:621-30.

62. Davis LK, Maltman N, Mosconi MW, Macmillan C, Schmitt L, Moore K, Francis SM, Jacob S, Sweeney JA, Cook EH. Rare inherited A2BP1 deletion in a proband with autism and developmental hemiparesis. Am J Med Genet A. 2012;158A:1654-61.

63. Werling DM, Geschwind DH. Sex differences in autism spectrum disorders. Curr Opin Neurol. 2013;26:146-53.

64. Iossifov I, O'Roak BJ, Sanders SJ, Ronemus M, Krumm N, Levy D, Stessman HA, Witherspoon KT, Vives L, Patterson KE, et al. The contribution of de novo coding mutations to autism spectrum disorder. Nature. 2014;515:216-21.

65. Sanders SJ, Murtha MT, Gupta AR, Murdoch JD, Raubeson MJ, Willsey AJ, Ercan-Sencicek AG, DiLullo NM, Parikshak NN, Stein JL, et al. De novo mutations revealed by whole-exome sequencing are strongly associated with autism. Nature. 2012;485:237-U124.

66. Kremer EJ, Pritchard M, Lynch M, Yu S, Holman K, Baker E, Warren ST, Schlessinger D, Sutherland GR, Richards RI. Mapping of DNA instability at the Fragile-X to a trinucleotide repeat sequence $P(C c g) N$. Science. 1991;252:1711-4.

67. Verkerk AJ, Pieretti M, Sutcliffe JS, Fu YH, Kuhl DP, Pizzuti A, Reiner O, Richards S, Victoria MF, Zhang FP, et al. Identification of a gene (FMR-1) containing a CGG repeat coincident with a breakpoint cluster region exhibiting length variation in fragile X syndrome. Cell. 1991;65:905-14.

68. Vincent A, Heitz D, Petit C, Kretz C, Oberle I, Mandel JL. Abnormal pattern detected in fragile-X patients by pulsed-field gel electrophoresis. Nature. 1991;349:624-6.

69. Lal D, Pernhorst K, Klein KM, Reif P, Tozzi R, Toliat MR, Winterer G, Neubauer $B$, Nurnberg $P$, Rosenow $F$, et al. Extending the phenotypic spectrum of RBFOX1 deletions: sporadic focal epilepsy. Epilepsia. 2015;56:e129-133.

70. Basu SN, Kollu R, Banerjee-Basu S. AutDB: a gene reference resource for autism research. Nucleic Acids Res. 2009;37:D832-836.

71. Lammert DB, Howell BW. RELN Mutations in autism spectrum disorder. Front Cell Neurosci. 2016:10:84

72. Bacon C, Schneider M, Le Magueresse C, Froehlich H, Sticht C, Gluch C, Monyer H, Rappold GA. Brain-specific Foxp1 deletion impairs neuronal development and causes autistic-like behaviour. Mol Psychiatry. 2015;20:632-9.

73. Yamasaki A, Kasai A, Toi A, Kurita M, Kimoto S, Hayata-Takano A, Nakazawa T, Nagayasu K, Shintani N, Hashimoto R, et al. Identification of the role of bone morphogenetic protein (BMP) and transforming growth factor-beta (TGF-beta) signaling in the trajectory of serotonergic differentiation in a rapid assay in mouse embryonic stem cells in vitro. J Neurochem. 2015;132:418-28.

\section{Submit your next manuscript to BioMed Central and we will help you at every step:}

- We accept pre-submission inquiries

- Our selector tool helps you to find the most relevant journal

- We provide round the clock customer support

- Convenient online submission

- Thorough peer review

- Inclusion in PubMed and all major indexing services

- Maximum visibility for your research

Submit your manuscript at www.biomedcentral.com/submit 